\title{
La séduction, l'abject et le corps féminin dans les romans de Maurice Henrie
}

\section{Kathleen Kellett-Betsos, Université Ryerson}

Né en 1936 à Rockland, l'auteur franco-ontarien Maurice Henrie offre dans sa fiction un mélange de réalisme et de fantastique qui donne lieu à une critique sociale percutante. Connu surtout pour ses nouvelles, Henrie a également publié trois romans qui révèlent un univers régi par l'angoisse existentielle. Son premier roman, Le balcon dans le ciel (1995), lui valut le Prix Trillium ${ }^{1}$. Dès le début du roman, le lien intertextuel avec La Chute d'Albert Camus est établi lorsque le narrateur, sociopathe charmant, reconnaît le livre de Camus entre les mains de sa prochaine victime. Le récit du narrateur, dont le style s'apparente à celui du personnage camusien Jean-Baptiste Clamence, retrace l'histoire d'une séduction où le corps féminin devient l'enjeu d'une lutte entre Éros et Thanatos. Le deuxième roman d'Henrie, Une Ville lointaine (2001), raconte une descente en enfer signalée par des renvois intertextuels à la Divine Comédie de Dante déjà analysés en détail dans un article fort pertinent de Michel Ouellette. Entre la ville francocanadienne de l'Espérance et la ville mystérieuse d'Escanaba, située d'un côté ou de l'autre de la frontière canado-américaine, Odette Désabrais cherche son mari Antoine qui l'a délaissée en faveur d'une quête transcendantale qu'elle comprend mal. Attachée à la vie terrestre par mille soucis domestiques, préoccupée à rejoindre le bien-aimé tant désiré, elle est également objet du désir chez les hommes qui l'entourent, dont surtout un jeune homme à la recherche de sa mère également disparue en quête d'Escanaba. Dans Le Chuchotement des étoiles (2007), un homme s'apitoie sur les efforts de sa femme mystérieusement vieillie de le séduire ; pour lui, le corps féminin vieillissant est un objet abject. Après la mort de sa femme bien-aimée, ce dernier aspire à se défaire des liens qui le rattachent à la terre, visant plutôt un refuge parmi les étoiles. Chez Henrie, la représentation de l'abject relève d'une équivalence établie entre la vie cérébrale au masculin et la vie corporelle au féminin. Il appert que l'œuvre d'Henrie participe de la «somotaphobie », phénomène dont la philosophe américaine Elizabeth Spelman, dans son article « Woman as body : Ancient and Contemporary Views », trace la manifestation depuis Platon : il s'agit d'une dévalorisation du corps humain par rapport à l'esprit. 
Comme le souligne Spelman, la tradition occidentale a toujours eu tendance à associer le corps surtout à la femme et l'esprit surtout à l'homme. L'homme jouit de ce que la critique féministe Patricia Yaeger appelle l'asomie, le principe de désincarnation ${ }^{2}$. Dans les trois romans de Maurice Henrie, trois images archétypales de la femme ressortent : la femme séduite et séduisante ; la mère et épouse avaleuse et accaparante ; et la vieillarde au corps menacé de désintégration et de putréfaction. Cette opposition entre le corps et l'esprit s'avère d'autant plus problématique que l'idée même de transcendance semble dépassée et interdite à cette époque où, aux yeux d'Henrie, les dieux sont disparus du ciel.

Les thèmes principaux de l'œuvre d'Henrie se rattachent autant à la notion de l'absurde chez Camus qu'à la cosmogonie chrétienne de Dante. Dans la société francocanadienne contemporaine, où les doctrines catholiques n'ont plus l'emprise sociale de naguère, l'individu moderne hésite à se fier à l'assurance d'un paradis à la fin de ses jours. Les romans d'Henrie mettent en vedette des personnages masculins qui recherchent désespérément un monde au-delà de la vie terrestre. Ce mépris de la vie ici-bas se manifeste par une focalisation sur l'abject où la fluidité des frontières entre soi et l'autre, entre l'humain et l'animal, entre la vie et la putréfaction de la mort, menace l'intégrité corporelle et psychique de l'être humain. Dans les romans à l'étude, cette conscience de l'impureté trouve son point focal dans la représentation du corps féminin comme abject ou potentiellement abject. Comme l'explique Julia Kristeva dans son essai Pouvoirs de l'horreur, «L'abject nous confronte, d'une part, à ces états fragiles où l'homme erre dans les territoires de l'animal. Ainsi, par l'abjection, les sociétés primitives ont balisé une zone précise de leur culture pour la détacher du monde menaçant de l'animal ou de l'animalité, imaginés comme des représentants du meurtre et du sexe » (20). Associée aux exigences de la reproduction de l'espèce, la femme chez Henrie est sujet et objet du désir charnel ; elle empêche toute tentative de transcendance. Plus particulièrement, dans Le Balcon dans le ciel, la femme séductrice et la femme reproductrice, corps séduisant et corps maternel avaleur, risquent constamment de se confondre. D'ailleurs, Kristeva rapproche le sentiment d'abjection aux difficultés de l'individuation par rapport au corps maternel : 
L'abject nous confronte, d'autre part, et cette fois dans notre archéologie personnelle, à nos tentatives les plus anciennes de nous démarquer de l'entité maternelle avant même que d'ex-ister en dehors d'elle grâce à l'autonomie du langage. Démarquage violent et maladroit, toujours guetté par la rechute dans la dépendance d'un pouvoir aussi sécurisant qu'étouffant. (20)

De la même façon, en tant que mère et épouse, la femme chez Henrie est perçue comme obstacle à l'individuation autant pour son mari que pour ses enfants. C'est ainsi qu'Odette Désabrais dans Une Ville lointaine doit éventuellement renoncer à retenir les membres de sa famille qui cherchent à fuir le bonheur familial qu'elle essaie de leur imposer. Enfin, sur le tard de sa vie, comme on le voit notamment dans Le Chuchotement des étoiles, la femme qui vieillit représente l'abject par excellence, incarnation du memento mori, la beauté féminine subissant les ravages de la détérioration menant à la mort.

\section{La femme séductrice}

C'est dans Le Balcon dans le ciel que l'image de la femme séductrice suscite une réaction d'attirance et de répulsion extrême, amenant le personnage principal à se défendre de l'abjection associée au corps féminin au moyen du meurtre prémédité, rupture sociale que Kristeva identifie comme acte abject :

Ce n'est donc pas l'absence de propreté ou de santé qui rend abject, mais ce qui perturbe une identité, un système, un ordre. Ce qui ne respecte pas les limites, les places, les règles. L'entre-deux, l'ambigu, le mixte. Le traître, le menteur, le criminel à bonne conscience, le violeur sans vergogne, le tueur qui prétend sauver... Tout crime, parce qu'il signale la fragilité de la loi, est abject, mais le crime prémédité, le meurtre sournois, la vengeance hypocrite le sont plus encore parce qu'ils redoublent cette exhibition de la fragilité légale. (12)

Les rapports ambigus entre le narrateur et le désir charnel font surface dans son adolescence passée dans un petit village francophone de l'Ontario. Le narrateur ambitieux voit la femme qui essaie de le séduire «comme un obstacle qu' on ne peut contourner » (39) : «Petite Gisèle d'autrefois, aux hanches maintenant larges et enveloppantes, qui porteraient un jour un enfant. Celui de quelqu'un d'autre » (39). Plus tard, à Ottawa, où il fait ses études pour éventuellement devenir avocat au gouvernement, lui et son ami d'enfance Jean-Pierre, ombre fidèle et colocataire, doivent faire face aux 
avances de leur propriétaire séductrice en kimono qui laisse révéler « une belle cuisse blanche et ferme » et même «le bout brunâtre d'un mamelon » (43). Heureusement, la logeuse en vient à conclure que les deux hommes sont en fait des amants et donc insensibles à ses charmes, croyance qu'ils tiennent à encourager. Par contre, lorsque le narrateur s'éprend de la belle et blonde Stéphanie, c'est aux dépens de son rapport avec Jean-Pierre. Son amour pour Stéphanie est féroce et possessif : «En fait, si depuis toujours je me reconnais la liberté d'aller où il me plaît, j'ai compris dès ce moment-là que ce privilège ne s'étendrait plus jamais à Stéphanie. Elle est entrée volontairement dans ma vie sans se rendre compte qu'elle n'en ressortirait jamais » (52). Quoique, selon lui, Stéphanie essaie de faire appel à son esprit, c'est plutôt par le corps qu'il s'engage avec elle ; il est attiré par « le parfum animal qui se dégageait de son corps » (54). À cet égard, le narrateur évoque la célèbre dichotomie entre corps et esprit : «Est-il d'ailleurs si certain que l'esprit doive toujours l'emporter sur le corps ? Surtout lorsqu'il s'agit d'amour. Je ne pense pas » (16).

La description que fait le narrateur de cette passion évoque la fusion pré-œdipale entre l'enfant et le corps maternel : «Je séjournais profondément dans son corps, aussi longtemps et aussi souvent que mes forces me le permettaient. Je l'habitais comme un fœtus. Je me nourrissais d'elle. Je me laissais même porter par elle. Nous mêlions le plus souvent possible les fluides de nos deux corps » (13). Ce manque d'individuation, ce brouillage des frontières entre soi et l'Autre, représente une menace que le narrateur se devra de combattre. Avec le temps, il s'endurcit contre son amante :

[...] mon amour pour Stéphanie prenait souvent la forme d'une lame tranchante. J'entrais dans sa chair comme un couteau dans un melon mûr. Je pénétrais dans son esprit comme un scalpel dans la chair vive. Malgré moi, des images de vivisection, de venaison, de charcuterie m'effleuraient à l'improviste, que je devais chasser en faisant un effort conscient, pénible même. J'en arrivais à des hallucinations où Stéphanie se transformait en un muscle géant, tout rouge, encore chaud de vie, toujours vibrant de sensations spasmodiques. (17)

La violence de cette description, cette manière de réduire la femme par synecdoque à un morceau de charcuterie, à « un muscle géant», manifeste la force de l'horreur qu'inspire l'abject chez cet homme qui voudrait rétablir les frontières entre lui et le corps féminin, le corps maternel. 
Le narrateur en vient à mépriser cette femme qui s'imagine l'emmener avec elle sur le «carrousel magique » (56) de ses rêves de bonheur domestique : «Petite femme centripète qui croyait avoir le cœur assez grand pour qu'on y mette le monde. Qui s'imaginait posséder l'univers entre ses deux jambes blondes » (64). Comme Gisèle, Stéphanie devient un obstacle à contourner. Derrière elle, le narrateur entrevoit un monde mystérieux, «[1]'univers des ombres diaphanes et prometteuses» (67). Le corps de Stéphanie devient transparent pour lui lorsqu'il s'acharne à voir l'écran magique qui lui semble apparaître derrière elle. Il invite Jean-Pierre à explorer ce nouveau monde désincarné avec lui mais celui-ci, délaissé par son ami d'enfance, a trouvé de nouveaux intérêts moins cérébraux tels que le jogging et, comme le découvrira éventuellement le narrateur, le corps à corps avec la belle Stéphanie. Ici, la femme séductrice représente une double menace : son corps empêche l'accès non seulement au monde transcendant de l'esprit mais aussi au domaine idéalisé de l'amitié au masculin, dont le narrateur a refoulé toute suggestion de pulsions homo-érotiques.

Déterminé à reprendre son trophée, le narrateur accepte de monter sur le «carrousel magique» du mariage. De femme séductrice, Stéphanie devient femme génitrice et l'abject ressurgit de nouveau dans le corps maternel sujet aux malaises et aux vomissements. On remarque la laideur de sa description lorsque le narrateur se dit insensible «à ses larmes, à son beau visage rougi et convulsé par les spasmes et les expectorations »(125). L'abjection est soulignée par les saignements apparemment spontanés de la femme : «C'est à cette époque que, pour la première fois, Stéphanie a commencé à perdre son sang. Oui, pour un rien, elle se mettait à saigner, surtout du nez et de la bouche, parfois aussi d'autres plaies sur son corps » (128). Le narrateur est conscient des trous dans son histoire et n'avoue pas directement la violence conjugale dirigée contre sa femme :

Il m'est arrivé souvent de la trouver le visage tuméfié ou même la lèvre fendue à l'intérieur, vis-à-vis des dents. Sans compter que des ecchymoses bleues ou violettes sont apparues un peu partout sur le corps, dans les endroits les plus improbables, comme si une volonté cachée s'exerçait contre elle et cherchait à lui faire du mal. J'étais sidéré de ce qui arrivait et j'avais le plus grand mal à regarder Stéphanie dans les yeux, surtout quand elle semblait souffrir plus qu'à l'ordinaire. (128-9) 
Lorsque le narrateur découvre l'infidélité continue de sa femme et de son ami JeanPierre, il fait couler leur sang dans son balcon dans le ciel.

En tant que symbole, le balcon se rapproche du monde obscur entrevu par le narrateur derrière la femme-obstacle, puisqu'il représente la même tentative de s'évader de la vie commune des mortels d'en bas. Par contre, si l'écran, «ce trou translucide » (86) «n'a pas tenu promesse », selon le narrateur, s'il est devenu plutôt « une sorte de gueule insatiable» qui «avalait» son monde familier (120), s'approchant donc de l'abject, le balcon offre à la fois la supériorité des hauteurs et le plaisir de vengeance dans une vision du monde où la moralité chrétienne n'a aucune emprise. Comme dans $L a$ Chute de Camus, le narrateur favorise les hauteurs, le balcon étant l'équivalent de «la cime du ciel hollandais » où Jean-Baptiste Clamence prend plaisir à s'imaginer jouer à Dieu le père et à dominer les autres êtres humains (Camus 149). Le narrateur fait la description du balcon à Janelle, sa nouvelle conquête et prochaine victime : «Mon balcon aussi est désert, silencieux et imprégné du parfum de dieux disparus. Une chapelle volante? Si vous voulez»(114). Lorsqu'il attire la femme séductrice sur le balcon, le meurtre qui s'ensuit n'est pas tout à fait inattendu pour le lecteur. Comme le narrateur entreprend à la fin du roman la séduction d'une autre femme «aux longues jambes blanches et aux cuisses minces, presque frêles »(145), on se rend compte de son intention de se venger de nouveau au moyen de la destruction de ce corps féminin qui représente un empêchement à la quête de transcendance.

Par contre, tout en associant le corps abject au féminin, Le Balcon dans le ciel ne présente pas l'assujettissement de la femme comme inévitable. Le narrateur profite d'une société où les femmes subissent la pression sociale d'être naïves et douces ; il dit carrément à Janelle que c'est sa faiblesse qui l'attire : «Je me roule en boule comme un chat heureux, sachant que vous ne me repousserez pas, que la bonté est chez vous faiblesse, malgré votre maquillage agressif et vos airs de grande chasseresse » (9-10). Selon lui, c'est la couleur rose, imposée aux femmes par la société, signe de faiblesse et de compromis, qui mène à leur chute :

Je suis partisan d'une révolte ouverte des femmes contre le rose, qui me semble une machination subtile et efficace des hommes pour les entretenir dans un état de perpétuelle disponibilité, d'où elles peuvent tomber, selon ce qu'ils ont déjà décidé dans leur cœur noir, d'un côté ou de l'autre. Le 
rose, cet esclavage à peine voilé, ce renoncement heureux à la volonté, ce recul instinctif devant le choix. Si j'étais de votre sexe, Janelle, je fonderais une association de femmes contre le rose, bien avant de me préoccuper de parité salariale. (27-28)

D'après cette rhétorique qui blâme la victime, si la femme est réduite à un corps à conquérir et à détruire, c'est qu'elle n'assume pas pleinement son agentivité potentielle. Ayant trouvé dans la quête transcendantale un leurre, le tueur profite de la naïveté des femmes pour essayer encore et encore d'expulser du haut de son balcon l'abject incarné par le corps féminin, déchiré et saignant.

\section{La femme-épouse avaleuse}

Si la femme séductrice et féconde du Balcon dans le ciel suscite des images de l'abject, dans Une Ville lointaine, à première vue, Odette Désabrais semble correspondre à l'épouse et à la mère parfaite, Orphée au féminin qui entreprend la descente aux enfers pour soustraire son bien-aimé du monde des morts. Dans son étude «Une Ville lointaine de Maurice Henrie : une descente en enfer postmoderne », Michel Ouellette signale les ressemblances entre la route vers Escanaba et la géographie cosmogonique de la Divine Comédie de Dante. Cet intertexte rejoint le contexte camusien et son évocation de l'absurde, comme le suggère ce commentaire du narrateur de La Chute : «Connaissezvous Dante ? Vraiment ? Diable. Vous savez donc que Dante admet des anges neutres dans la querelle entre Dieu et Satan. Et il les place dans les Limbes, une sorte de vestibule de son enfer. Nous sommes dans le vestibule, cher ami » (89). Remplie d'espoir au début de sa quête, Odette Désabrais en vient à comprendre que la fuite de son mari est une tentative de sortir de « ce vestibule de l'enfer » qu'est la vie.

Pour sa part, François Paré a examiné le sentiment de malaise dans Une Ville lointaine en fonction de la migration des Canadiens-français vers l'ouest et vers les ÉtatsUnis $(2006,2007)$. En fait, le phénomène de déterritorialisation ${ }^{3}$ chez le minoritaire peut expliquer en partie cette angoisse existentielle. L'article d'Ouellette se fonde également sur le contexte socio-historique puisqu'il note que la ville de l'Espérance, d'où viennent Odette et son mari, s'appelait autrefois Sainte-Anne de l'Espérance et que l'utilisation de la forme raccourcie souligne la sécularisation de cette ville dont la raison d'être est surtout économique. Ouellette remarque aussi que le mari d'Odette travaille pour 
l'agence de recouvrement Dixon Collection, ce qui suggère la puissance capitaliste angloaméricaine (65-66). Antoine Désabrais a succombé au désespoir engendré par un monde sans but autre que l'accumulation des richesses. Devant ce vide existentiel, les valeurs conjugales et familiales de sa femme ne lui suffisent plus.

Fidèle à son mari disparu, Odette entreprend sa quête sans se laisser séduire par les regards admirateurs des hommes. Quoique flattée par l'admiration masculine, elle refuse les avances du jeune Jacques Saint-Jacques, sachant que son attirance pour elle se rattache à son désir de retrouver la figure maternelle perdue, sa mère étant partie depuis un certain temps en route vers Escanaba sans mot d'explication à son fils. Mélanie, fille d'Odette, cherche même à faire concurrence à sa mère en essayant de séduire SaintJacques mais elle doit admettre que celui-ci ne s'intéresse qu'à Odette, car Odette est la mère par excellence dans la mesure où elle cherche à assurer le bien-être des siens. C'est exactement ce que Mélanie lui reproche en lui demandant ce qu'elle ferait si jamais elle trouvait son mari. Est-ce qu'elle essaierait de le ramener à « [son] petit bonheur étroit »? Est-ce qu'elle le renverrait à la «prison » de sa vie conventionnelle ? (273) La mère et la fille entrevoient Antoine assis tranquillement dans un train qui roule sans fin, un train nommé Paradisum, allusion à la cosmogonie de Dante (Ouellette 61). En décidant de rester dans le train qui s'achemine vers la mer, Antoine choisit lui-même la fusion préœedipale quoiqu'il préfère une matrice désincarnée, éloignée de la nature animale: «Antoine regarde la mer illuminée par sa propre lumière, la mer qui se reflète dans l'air incertain du jour qui naît ou qui meurt, c'est selon. Antoine entre dans la mer, le refuge par excellence » (217).

Le rapport entre la descente aux enfers et l'abject se poursuit avec le lieu symbolique des deux animaleries, l'une à l'Espérance l'autre aux alentours d'Escanaba, où travaille le commis Antoine. Selon Ouellette, «l'animalerie pourrait représenter la porte d'entrée de l'enfer», interprétation suggérée par les trois races de chiens mentionnées par le commis et qui « rappellent Cerbère, le chien à trois têtes, gardien de la porte des enfers » (61). Ayant remarqué la curieuse liberté de cet homme de passer librement d'une ville à l'autre, Odette l'interroge sur Escanaba et le sort possible de son mari. Le commis se rapproche du mari d'Odette par son angoisse existentielle : 
Tout ce que je peux vous dire, c'est que j'éprouve du soulagement à changer de ville, à changer de boutique, à changer de grisaille. Et si je m'intéresse à votre mari, c'est que je n'en peux plus, moi non plus. C'est que moi aussi je cherche à tromper l'ennui, à me soustraire à la terrible routine, à fuir l'abominable dissolution à laquelle nous sommes tous condamnés. [...] Madame Désabrais, je suis excédé de voir à quel point mon existence est fortuite, combien je suis une créature du hasard, le résultat d'une collision biologique. (251-252)

Comme le remarque Ouellette, le commis est le seul personnage qui se déplace entre L'Espérance et Escanaba par avion : «Sa voie est céleste et non terrestre » (60). Plutôt rattachée à la terre, Odette se contente de sa Jeep pour traverser le labyrinthe qu'est la route vers Escanaba. Elle ne renie ni L'Espérance ni la vie terrestre. Elle accepte le défi de vivre dans un monde où l'être humain est le produit d'une «collision biologique » plutôt que de la providence divine. Par contraste avec la mère de Jacques Saint-Jacques qui se contente de rester à l'Hôtel Clarendon à la périphérie d'Escanaba où elle sombre dans une amnésie apaisante, ayant sans doute bu des eaux de Léthé, Odette choisit de retourner auprès de son jeune fils à L'Espérance, malgré la difficulté de naviguer le labyrinthe autour d'Escanaba: «Je repars pour L'Espérance. Je repartirai pour L'Espérance aussi longtemps et aussi souvent qu'il le faudra » (290).

Quoique d'une façon moins directe que Le Balcon dans le ciel, Une Ville lointaine met en relief les rapports entre la femme et la nature animale de l'être humain. C'est Odette qui attire le regard masculin désirant : de la part de Jacques Saint-Jacques ; de Norbert, employé de l'hôtel Clarendon ; d'Antoine Beauvais, éditeur du journal L'Écho métropolitain qui admire sa voix «chaude et érotique » (114), entre autres. Alors que son mari traverse les limbes en route vers la mer, Odette rêve de l'avoir dans ses bras et se réveille en découvrant un amant qui s'enfuit dans le couloir et laisse l'odeur du sperme dans son lit. Elle affirme sa nostalgie de la fusion corporelle et émotive avec son mari en l'interpellant : «Moi, je ne t'ai pas oublié. Je suis devenue un trou, un vide qui a la forme et le volume de ton corps. Jamais personne d'autre ne pourra le combler tout à fait, ni exactement. De sorte que j'aurai toujours faim de toi, mon amour » (235). Par contre, son épiphanie est de se rendre compte qu'elle ne pourra pas retenir contre leur gré ceux qu'elle aime :

Je me suis trop longtemps cru un droit, un devoir de chercher ceux qui s'en allaient, de les poursuivre de mon affection, de les relancer où qu'ils 
soient. Maintenant, j'accepte leur départ [...] Surtout, je leur reconnais à eux le droit de nous quitter, de ne plus rester là où toi et moi nous sommes encore. Dans ce monde incohérent, illogique, sautillant dans lequel nous vivons. Où tout nous crie que nous sommes des accidents, des excédentaires. Un monde si peu désirable, si peu accueillant que je me demande pourquoi nous voulons tant y rester. (285)

Comme l'indique Kristeva, la passion maternelle rend difficile le renoncement à la fusion pré-œdipale entre la mère et l'enfant : «La difficulté d'une mère à reconnaitre (ou à se faire reconnaître par) l'instance symbolique - autrement dit ses embarras avec le phallus que représente son père à elle ou son mari - n'est évidemment pas de nature à aider le futur sujet à quitter l'auberge naturelle » (20). Figure maternelle, Odette finit par accepter la mission de faciliter la transition de ceux qui cherchent à quitter «l'auberge naturelle » du corps maternel sans renier elle-même la vie terrestre et l'abject : «J'ouvre tout grand les bras - avec tendresse, sans la moindre amertume -, j'ouvre tout grand les bras à l'incohérence et à l'horreur de cette terre » (285). Une telle renonciation suggère une transformation de l'abject en sublime.

\section{Le corps féminin et la déchéance}

La déchéance du corps par la dégénérescence physique et éventuellement la mort est le thème central du Chuchotement des étoiles. La reprise du nom Odette pour la femme du protagoniste Pierre implique qu'il s'agit d'un nouvel aspect de la femme archétypale. Comme Antoine, Pierre refuse les ennuis du monde contemporain. Il se réfugie dans une maison parmi les astres, loin de cette ville de fonctionnaires où il travaillait autrefois et où ses deux enfants font carrière. Il se reconnaît une intelligence et une perspicacité supérieures à celles de sa femme : «Bien sûr, Odette avait des limites évidentes, visibles même. Elle était incapable des envolées lyriques et métaphysiques où Pierre excellait, ni des états supérieurs qu'il prétendait atteindre » (36). À la différence de sa femme, Pierre perçoit des phénomènes surnaturels inexplicables : un masque en laiton qui le regarde du plafond, la voix d'un enfant qui lui demande au téléphone s'il est «le créateur du ciel et de la terre » (32) et, surtout, le vieillissement rapide de sa femme dont elle-même ne se rend pas compte. Face à son travail de rédiger un discours pour le Ministre de la Culture, Pierre ressent la nausée, ce signe de la lutte avec l'abject, comme le souligne Kristeva dans ce passage: «Dégoût d'une nourriture, d'une saleté, d'un 
déchet, d'une ordure. Spasmes et vomissements qui me protègent. Répulsion, haut-lecœur qui m'écarte et me détourne de la souillure, du cloaque, de l'immonde. Ignominie de la compromission, de l'entre-deux, de la traîtrise. Sursaut fasciné qui m'y conduit et m'en sépare » (10). Pierre est convaincu qu'Odette ne pourrait jamais comprendre ses pensées : «Elle refuserait d'admettre qu'on puisse être malade de penser. Et qu'on puisse en vomir » (65). Il déplore le monde politique où les politiciens prononcent des paroles écrites par autrui. C'est pourtant lui qui a accepté ce travail qu'il trouve indigne même s'il reste anonyme en tant que rédacteur : «Seul à savoir qu'il vivait en contradiction avec lui-même, son esprit et ses doigts collaborant à un travail que tout son être réprouvait»(118). Le dégoût de «la vie secrète des grands bureaucrates », pour reprendre le titre de l'ouvrage satirique d'Henrie (1989), représente pour Pierre une forme de l'abject liée à la rupture de l'ordre sociale. Ce sentiment de répulsion s'exprime donc par le vomissement, spasme musculaire qui représente la violence physique du refus.

Le corps vieillissant d'Odette suscite chez Pierre l'horreur associée à l'abject. Le corps se disloque, la salive coule, les hanches s'ankylosent. Les cheveux grisonnent au point où Odette met « une perruque auburn frisée » (107) pour les cacher à son mari qui ne supporte pas de voir un seul cheveu gris. Odette n'est pas consciente de sa déchéance accélérée : elle se maquille, elle jette à son mari des regards qui se veulent séducteurs, elle demande un baiser alors que sa bouche est flasque et édentée : « Le temps lui abîmait son Odette au point de la rendre méconnaissable. Il l'endommageait dans sa chair, ce qui était facilement reconnaissable, et aussi dans son esprit, ce qui était plus subtil et plus insidieux » (109). Par comparaison, Pierre se sent assez en forme, comme si sa femme mourait à ses dépens, comme s'il projetait sur sa femme sa propre peur de désintégration corporelle. Devant ce regard mâle, la femme en vient à représenter le memento mori, le rappel de la fragilité de la vie terrestre. Pierre remarque que même dans la mort, le corps d'Odette continue à vieillir : «Ses cheveux blancs commençaient à se séparer de son crâne, les os des pommettes et du menton menaçaient de percer la peau du visage et les taches duveteuses de la décomposition, roses et vertes, mouchetaient déjà son front et ses joues. Même dans la mort, Odette continuait de vieillir à un rythme accéléré » (129). Il déplore personnellement les indignités de la vieillesse et surtout le fait que les proches en 
soient témoins : « À ce sujet, une image lui revenait constamment à l'esprit. Celle de ces organisations terroristes qui contraignent la mère et les enfants à assister à la torture et à l'exécution du père » (108). Sa nausée devant le vieillissement et la mort prématurés de sa femme provient de cette indignation contre la dégénérescence du corps, surtout celle du corps féminin qui a perdu ses pouvoirs de séduction.

Après la mort d'Odette, Pierre emménage en ville pour être auprès de son fils et de sa fille. Comme Stéphanie dans Le Balcon dans le ciel, Odette a séduit son homme et l'a entraîné sur le «carrousel magique » de la vie domestique. Comme le commis Antoine dans Une Ville lointaine, Pierre est horrifié de mener une vie soumise aux accidents de la biologie : «Il refusait de participer plus longtemps à cette succession infinie de générations humaines, à ce perpétuel recommencement sans but où les spermatozoïdes se transforment en fœtus, les fœtus en enfants, les enfants en géniteurs, les géniteurs en fœtus, avant de devenir eux-mêmes vieillards, puis cadavres » (61). La notion de trouver une immortalité quelconque à travers la progéniture ne lui dit rien. D'ailleurs, son fils avocat participe lui aussi à des compromissions du monde du travail mais sans en souffrir. Sa fille Paule est douce comme sa mère mais sa compagnie occasionnelle ne suffit pas à faire reculer l'angoisse.

La ville sans nom où habitent les enfants de Pierre, est une caricature du siège gouvernemental qu'est Ottawa. Elle représente pour lui un lieu déchu, surtout après la vie dans la «maison interstellaire » (123) qu'il a dû quitter. C'est de cette ville que lui parviennent les travaux qui lui donnent la nausée, que ce soit la rédaction d'un discours politique ou la traduction d'un document sur les munitions utilisant l'uranium appauvri. Les images de l'abjection le suivent à la ville lorsqu'il s'arrête à une espèce de labyrinthe, une fête foraine remplie de scènes de dégradation humaine : une femme qui se met à genoux en public pour faire jouir un banquier blasé, un écrivain qui écrit sous la dictée des idées reçues en aspirant à la gloire, un enfant qui poignarde une victime devant une foule appréciatrice. Pierre est réprimandé pour avoir cédé au besoin physique de vomir, acte qui brouille les frontières entre le corps et le monde extérieur. Enfin libéré du labyrinthe, il entreprend de retourner à son « habitation suspendue dans l'espace » (123). Par contraste avec la mort physique et dégradante d'Odette, Pierre s'enfuira parmi les étoiles, libéré de ce corps imparfait, forme de transcendance qui rappelle le voyage 
d'Antoine Désabrais vers la mer dans Une Ville lointaine. Si la femme-victime, réduite à un corps disloqué et fragmenté, meurt chez Henrie sous le signe de la synecdoque, l'homme au corps intact rejoint la mort tranquillement par la métaphore, à l'exception bien sûr de Jean-Pierre, infidèle au pacte de l'amitié masculine. Les personnages masculins chez Henrie s'en tirent donc sans connaître la déchéance de la dégénérescence physique alors que les femmes sont sujettes à l'abjection et rappellent aux protagonistes mâles la fragilité du corps humain.

\section{Conclusion : Angoisse et agentivité dans un monde absurde}

Dans les romans d'Henrie, la lourdeur corporelle des femmes sert à évoquer la condition terrestre de l'humanité et l'impossibilité pour l'homme d'expérimenter la transcendance, sauf à travers l'utopie des mondes interstellaires ou mythiques. La vie de la femme y implique le compromis et la domesticité, l'attirance vers le bas qui contraste avec l'asomie, la désincarnation relative des hommes. On pourrait y voir un rappel du passé janséniste des Canadiens français, une certaine vision ascétique, héritage d'une société autrefois imbue du catholicisme. Que l'on pense au poète tourmenté, Hector de Saint-Denys Garneau, qui disait pensivement dans son Journal : « À travers ma vie, l'impression que l'innocence était refoulée de plus en plus de bas en haut. Un désir en même temps de n'avoir rien à faire avec la partie corrompue, la partie sans lumière [...] Ainsi, durant l'adolescence, une sorte de désir que mon corps finisse à la ceinture » (336337). Dans les romans d'Henrie, la cosmogonie chrétienne ne vient plus offrir du réconfort. Le sentiment de déterritorialisation chez ses personnages franco-canadiens minoritaires, la fluidité de l'espace franco-canadien mal défini, la corruption de la vie politique suggère que, chez Henrie, la ferveur nationaliste ne constitue pas non plus une source d'inspiration.

À l'instar de Camus, Henrie crée un univers romanesque marqué par un sentiment de l'absurde. Sans espoir de transcendance, l'être humain est guetté par la déchéance et la mort. Par contre, bien que la femme, objet du regard masculin, soit le point focal pour les soucis existentiels portés sur l'abject, il faut admettre que chez Henrie c'est aussi la femme qui a le potentiel de faire face à l'absurde et de manifester son agentivité, soit en choisissant de rejeter l'illusion de la vie en rose comme le narrateur encourage Janelle à 
faire, soit en fonçant de l'avant comme Odette Désabrais à travers un labyrinthe infernal. Si l'on peut trouver une étincelle d'héroïsme dans l'œuvre d'Henrie, c'est sûrement dans Une Ville lointaine où Odette Désabrais reste fidèle à l'amour qu'elle porte aux siens, au point même de les abandonner lorsqu'ils le lui demandent. Surmonter l'angoisse existentielle pour se définir par ses actes, même si sa quête semble inutile, préférer le souvenir à l'oubli apaisant : voilà la quête absurde de cette héroïne.

\section{Ouvrages cités}

Camus, Albert. La Chute. Paris : Gallimard, 1956.

Guattari, Félix et Gilles Deleuze. Kafka : pour une littérature mineure. Paris : Minuit, 1975.

Henrie, Maurice. Le Balcon dans le ciel. Sudbury : Prise de parole, 1995.

---. Le chuchotement des étoiles. Sudbury : Prise de parole, 2007.

---. Fleurs d'hiver ; Entre l'essai et la nouvelle. Sudbury : Prise de parole, 1998.

---. La Vie secrète des grands bureaucrates. Hull : Asticou, 1989.

---. Une Ville lointaine. Québec : L'Instant même, 2001.

Kristeva, Julia. Pouvoirs de l'horreur. Paris : Seuil, 1980.

Melançon, Johanne. «Maurice Henrie : un regard lucide sur soi et sur le monde ». Vers une étude de la nouvelle franco-ontarienne. Dir. François Paré. Virages 24 (automne 2003) : 21-35.

Ouellette, Michel. «Une Ville lointaine de Maurice Henrie: une descente en enfer postmoderne ». Revue du Nouvel-Ontario 31 (2006) : 55-73.

Paré, François. Le fantasme d'Escanaba. Montréal : Nota bene, 2007.

---. «Une Ville lointaine de Maurice Henrie ou le Chemin des disparus ». Romans de la route et voyages identitaires. Dir. Jean Morency, Jeanette den Toonder et Jaap Lintvelt. Montréal : Nota bene, 2006 : 175-189.

Saint-Denys Garneau, Hector de. Journal. Montréal : Bibliothèque Québécoise, 1996.

Spelman, Elizabeth. «Woman as body: Ancient and Contemporary Views ». Feminist Studies 8: 1 (printemps 1982) : 109-131. 
Yaeger, Patricia. "The Father's Breasts ». Refiguring the Father : New Feminist Readings of Patriarchy. Dir. Beth Kowaleski-Wallace et Patricia Yaeger. Carbondale et Edwardsville : Southern Illinois UP, 1989: 8-21.

\section{NOTES}

${ }^{1}$ Johanne Melançon indique qu'Henrie lui-même n'identifie pas Un Balcon dans le ciel comme un roman (21-22). Dans Fleurs d'hiver (1998), Henrie dit que: «[...] il est arrivé à un éditeur, pour des raisons de marketing, d'appeler roman un de mes livres, qui était en fait un recueil de nouvelles et, dans un autre cas, d'appeler aussi roman une seule nouvelle plus longue qu'à l'ordinaire » (27). La distinction entre un roman et une longue nouvelle n'est pas toujours évidente mais ici l'étiquette qui cherche à vendre l'œuvre sert aussi à en guider la lecture.

${ }^{2}$ Voir Patricia Yaeger : « [...] if somatophobia, or fear of the body's fleshliness and mutability characterizes our conflicts with women's bodies, then asomia, or bodilessness, characterizes our way of describing and thinking about the father $\gg(9)$.

${ }^{3}$ Voir Félix Guattari et Gilles Deleuze sur le rôle de la déterritorialisation dans les littératures dites mineures. 